\title{
论 文量子精密测量专题
}

\section{三电子原子 ${ }^{6} \mathrm{Li} \mathrm{D}$ 线跃迁绝对频率的精密测量}

\author{
芮扬，武跃龙，李睿，武海斌* \\ 华东师范大学, 精密光谱科学与技术国家重点实验室, 上海 200062 \\ *联系人, E-mail: hbwu@phy.ecnu.edu.cn
}

收稿日期: 2020-12-07; 接受日期: 2021-02-23; 网络出版日期: 2021-05-19

国家自然科学基金(编号: 11925401, 11734008)和国家重点基础研究发展计划(编号: 2017YFA0304201)资助项目

\begin{abstract}
摘要 在 ${ }^{6} \mathrm{Li}$ 冷原子系综中, 本团队发展了弱探测光和强本地光差拍探测的技术, 在极低探测光功率密度的 条件下 ( $<10^{-3}$ 饱和光强), 实现了达到光子散粒噪声极限的光谱测量. 基于高精度光学频率梳的频率稳定性 传递链, 将探测光锁到光梳上, 精确测量了 ${ }^{6} \mathrm{Li}$ 原子的 $\mathrm{D}$ 线跃迁频率, 得到不确定度优于 $1 \mathrm{kHz}$ 的 ${ }^{6} \mathrm{Li} \mathrm{D}_{1}$ 线跃迁 的绝对频率, 比当前最高精度的测量提高了一个量级. $2 \mathrm{P}$ 能级精细结构䢃裂和 $2 \mathrm{P}_{1 / 2}$ 能级超精细结构䢃裂分 别为 10052.7804(18)和26.1031(14) MHz, 与最近的相对论修正及量子电动力学修正的理论计算相符合. 本团 队的测量结果给更高精度的理论计算提供了实验基准, 同时也有助于解决之前不同实验测量的差异.
\end{abstract}

关键词冷原子, 绝对频率测量, 散粒噪声极限

PACS: 32.10.Fn, 42.62.Eh, 37.10.De, 42.50.Ar

\section{1 引言}

近年来, 锂原子的高精度光谱学在理论和实验 上都引起了人们广泛的关注 ${ }^{[1-5]}$. 中性锂原子核外 只有三个电子, 具有相对简单的能级结构, 可以在理 论上进行精确计算, 得到高精度的理论预测结果. 在 实验上通过精密光谱测量的方法确定能级结构, 可 以对已有的相关理论模型进行检验和修正, 并有望 发现新的物理模型.

近30年来, 少电子原子体系的精密计算取得 了巨大的研究进展. 通过Drake, Yan, Pachucki以及 Korobov等理论工作者的大量开创性工作 ${ }^{[6-12]}$, 氢原 子、氦原子和锂原子等少电子原子体系能级计算得
到了迅猛发展. 与此同时, 在实验测量方面, 氢原子、 氦原子光谱测量也取得了很大进展 ${ }^{[13-18]}$.

原子跃迁谱线的精密测量可以验证相对论 和量子电动力学(Quantum Electrodynamics, QED). 原子结构中相对论效应的计算通常是通过直接 使用Dirac方程进行的, 例如相对论组态相互作 用 ${ }^{[9]}$ 、多体微扰理论 ${ }^{[19]}$ 、相对论耦合簇 ${ }^{[20]}$ 或多 组态Dirac-Fock方法 ${ }^{[21]}$. 对于少电子系统, 更加精确 的计算方法是对精细结构常数中的能量进行级数展 开, 对高阶项进行修正. 该方法包含了相对论和量 子电动力学的贡献, 因为每次修正都可以用具有非 相对论波动函数的算子期望值表示. 通过使用显式 相关的基函数, 可以非常精确地求解少电子系统的

引用格式: 芮扬, 武跃龙, 李睿, 等. 三电子原子 ${ }^{6} \mathrm{Li} \mathrm{D}$ 线跃迁绝对频率的精密测量. 中国科学: 物理学 力学 天文学, 2021, 51: 074209

Rui Y, Wu Y L, Li R, et al. Precise absolute frequency measurement of three-electron ${ }^{6} \mathrm{Li}$ atom D-line transitions (in Chinese). Sci Sin-Phys Mech Astron, 2021, 51: 074209, doi: 10.1360/SSPMA-2020-0483 
非相对论Schrödinger方程. 但实现高精度的相对论 和QED修正, 需要计算与电子间距的反幂相关的复 杂积分. 对于锂原子, 目前 $2 \mathrm{P}$ 精细结构䢃裂的最精 确计算已达到 $\mathrm{m} \alpha^{6}$ 和 $\mathrm{m} \alpha^{7} \operatorname{In} \alpha$ 修正 ${ }^{[11,12]}$.

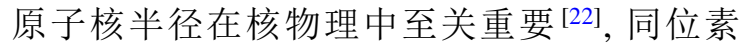
位移的精密测量对原子核半径的标定有巨大的帮 助. 尽管对于稳定同位素 ${ }^{6} \mathrm{Li}$ 和 ${ }^{7} \mathrm{Li}$ 的核半径大小的精 度可以达到 $1 \%$ 水平 ${ }^{[23]}$, 但对于不稳定的同位素 ${ }^{8} \mathrm{Li}$, ${ }^{9} \mathrm{Li}$ 和 ${ }^{11} \mathrm{Li}$, 其原子核半径却仍然不清楚. 除了传统 的核散射方法外, 还可以通过同位素位移实验测量 和理论计算相比较的方法研究核电荷的分布, 从而 确定核半径的大小. 尽管有限的核电荷分布对原 子能级有影响, 但是Drake ${ }^{[24]}$ 指出, 对于选定的跃迁, 如果对同位素位移中所有与质量相关的贡献都可 以计算得足够精确, 则实验和理论之间的差异就是 由核电荷半径的差异所引起的. 轻原子的QED项 在大小上可与核半径大小的一阶修正量相比拟, 且 与核质量无关, 因此它能在很大程度上与计算得出 的同位素位移相抵消. 因此, 该方法的重要性在于 可以不需要QED修正来确定核半径. 该方法已成 功应用于 ${ }^{4} \mathrm{He}-{ }^{3} \mathrm{He}^{[22,25]}$ 和 ${ }^{7} \mathrm{Li}^{6}{ }^{6} \mathrm{Li}^{[26]}$ 的研究, 所确定 的 ${ }^{3} \mathrm{He}$ 和 ${ }^{6} \mathrm{Li}$ 的均方根半径不但与核散射数据非常吻 合, 而且还大大提高了精度.

锂原子的精密光谱测量还导致了中子晕的发 现 ${ }^{[27-29]}$. 许多已知轻元素的稳定核心会影响弥散 在外部的中子的分布 ${ }^{[30]}$. 在锂的同位素中, 最著名 的是具有两个晕中子的 ${ }^{11} \mathrm{Li}$, 它由 ${ }^{9} \mathrm{Li}$ 的核与核外包 围的两个松散结合的中子共同组成 ${ }^{[31]} .{ }^{11} \mathrm{Li}$ 的质量 半径虽然已经从核的散射截面测量中确定 ${ }^{[27]}$, 但由 于两个晕中子对核电荷半径有影响, 所以不能仅用 核模型来确定核电荷半径. 因此, 关于晕中子是否 影响 ${ }^{9} \mathrm{Li}$ 核中质子分布的问题仍未解决. 对于有两 个晕中子 ${ }^{6} \mathrm{He}$ 的核电荷半径的测量 ${ }^{[32]}$ 表明, 它的晕 是绕 ${ }^{4} \mathrm{He}$ 核的中子形成的. ${ }^{6} \mathrm{He}$ 的核心是强结合的粒 子, 模型 ${ }^{[33]}$ 计算其核心仅比 ${ }^{4} \mathrm{He}$ 增加 $4 \%$. 相比之下, ${ }^{11} \mathrm{Li}$ 中的 ${ }^{9} \mathrm{Li}$ 核心是“较软”的, 晕中子与 ${ }^{9} \mathrm{Li}$ 核心之间 的相互作用可能会大大极化核心. 将实验测量结果 与核结构计算的结果进行比较, 将有助于更好地了 解中子滴线附近的核结构.

锂的高精度光谱学的发展为这些理论提供了
基准, 可用于确定核半径和测量精细结构常数, 从 而在更高的精度上检验基本物理定律. 与此同时, 已经有许多实验测量了 ${ }^{6} \mathrm{Li}$ 和 ${ }^{7} \mathrm{Li}$ 的 $\mathrm{D}_{1}$ 和 $\mathrm{D}_{2}$ 线的精细 结构䢃裂和超精细结构䢃裂以及同位素位移 ${ }^{[34-43]}$. 常见的测量方法是在热原子束中测量由激光激发 的苂光谱. 这种方法得到的数据为当前三电子模型 的最精确理论计算提供了重要的对比. 但这些实 验结果本身就有差异, 特别是在锂的 $\mathrm{D}_{1}$ 跃迁的同位 素位移测量中差异明显. Das和Natarajan ${ }^{[36]}$ 测量了 $2 \mathrm{P}_{J} \rightarrow 2 \mathrm{~S}$ 跃迁的同位素位移, 他们获得的精度对于 $\mathrm{D}_{1}$ 线 $(J=1 / 2)$ 为 $3.9 \mathrm{ppm}$, 对于 $\mathrm{D}_{2}$ 线 $(J=3 / 2)$ 为 $6.8 \mathrm{ppm}$. 后来Sansonetti等人 ${ }^{[42]}$ 测量 $2 \mathrm{P}_{J} \rightarrow 2 \mathrm{~S}$ 跃迁的同位素 位移, 他们达到的精度对于 $\mathrm{D}_{1}$ 线是 $0.9 \mathrm{ppm}$, 对于 $\mathrm{D}_{2}$ 线是 $29 \mathrm{ppm}$. 同时他们的测量值相互有偏差, $\mathrm{D}_{1}$ 线 同位素偏移测量值大约相差 $12 \sigma, \mathrm{D}_{2}$ 线的同位素偏 移测量值大约相差 $15 \sigma$. Wang等人 ${ }^{[12]}$ 的最新理论 计算同位素偏移和Sansonetti等人 ${ }^{[42]}$ 比较, $\mathrm{D}_{1}$ 线的 理论预测和实验测量相差 $180 \sigma, \mathrm{D}_{2}$ 线的理论预测和 实验测量相差 $60 \sigma$. 实验结果的不一致以及实验与 理论之间的差异仍然存在, 所以需要发展更高精度 的锂原子光谱以更精确地测量其频率.

\section{2 实验测量}

与以往在热原子束中测量不同, 本实验测量 $3^{6} \mathrm{Li}$ 冷原子的吸收光谱. 在冷原子中, 低温可以 极大地抑制一阶多普勒展宽, 并且二阶多普勒偏 移可以忽略. 同时, 在小跃迁几率处提高原子团 的光学密度, 从而获得较高信噪比的谱线. 结合 光学频率梳及差拍探测技术, 实现了光子散粒噪 声极限的谱线测量. 本团队 ${ }^{[44]}$ 精确测量了 ${ }^{6} \mathrm{Li} \mathrm{D}$ 线 跃迁的绝对频率、精细结构䢃裂和 $2 \mathrm{P}$ 精细结构䢃 裂, 并获得了不确定度低于 $1 \mathrm{kHz}$ 的 $\mathrm{D}_{1}$ 线跃迁频率. ${ }^{6} \mathrm{Li}$ 的 $2 \mathrm{P}_{1 / 2}$ 超精细结构䢃裂和 $2 \mathrm{P}$ 精细结构䢃裂分别 为26.1031(14)和10052.7804(18) MHz, 这与最新理 论计算相吻合.

\section{1 冷原子的制备}

本团队 ${ }^{[45,46]}$ 在超高真空中 $\left(\right.$ 近 $10^{-12}$ Torr $)$ 采用标 
准的磁光阱(Magneto-optical Trap, MOT)技术, 用三 对正交的圆偏光和磁阱俘获原子, 所采用的能级结 构以及每支光的频率失谐如图1所示, 冷却光和再 泵浦光的失谐为 -36 和 $-24 \mathrm{MHz}$, 光强分别为 27 和 $9 \mathrm{~mW}$, 光斑大小均为直径 $2 \mathrm{~cm}$. 塞曼光的失谐为 $-220 \mathrm{MHz}$, 光强为 $100 \mathrm{~mW}$, 偏振为圆偏光, 由透镜 将塞曼光汇聚至加热炉处. 磁场由一対反亥线圈 提供, 原子处的磁场梯度为 $10 \mathrm{Gauss} / \mathrm{cm}$. 通过装 载、冷却阶段, 可以获得温度为 $300 \mu \mathrm{K}$, 原子数约 为 $10^{8}$ 个的冷原子团.

由于 ${ }^{6} \mathrm{Li} 2 \mathrm{P}_{3 / 2}$ 态能级分裂为 $4.6 \mathrm{MHz}$, 小于自然 线宽 $5.8 \mathrm{MHz}$, 能级不可完全分辨, 没有通常意义上 的有效亚多普勒冷却机制. 为了使原子获得更低 的温度, 本团队发展了 $\mathrm{D}_{1}$ 线的灰色黏胶冷却 ${ }^{[47]}$ 技 术. 西西弗斯冷却(Sisyphus Cooling)和速度选择的 相干布居俘获(Velocity-Selected Coherent Population Trapping)两者的冷却效应在灰色黏胶冷却中同时 发挥效应. 在灰色黏胶冷却中两束蓝失谐的光将原 子缀饰出亮态和暗态. 对打的偏振光在空间上产生 偏振梯度, 使亮态在不同的空间位置产生不同的光 致失谐, 而暗态不耦合. 速度较高的原子才能从暗 态跃迁到亮态, 而处在亮态的原子在爬坡过程中损 失动能, 爬到势能高处后将通过双光子过程落回暗 态, 从而不断地损失动能, 最终将低速的原子贝禁 在暗态. 实验中 $\mathrm{D}_{1}$ 线冷却光路和MOT光路重合, 冷 却光和再百浦光频率如图2所示, $\mathrm{D}_{1}$ 线冷却光的单 光子失谐为 $-30 \mathrm{MHz}$, 双光子失谐为 $0 \mathrm{MHz}$. 原子 处 $\mathrm{D}_{1}$ 线冷却光的光束直径为 $5.6 \mathrm{~mm}$, 单支冷却光的 光功率为 $10 \mathrm{~mW}$, 再洜浦光的光功率为 $1 \mathrm{~mW}$. 作用 $1.5 \mathrm{~ms}$ 的 $\mathrm{D}_{1}$ 冷却光后, 最终获得了温度为 $50 \mu \mathrm{K}$, 原 子数约为 $5 \times 10^{7}$ 个的冷原子团 ${ }^{[48]}$.

\section{2 背景磁场的精确标定}

磁场会带来塞曼䢃裂和塞曼频移, 为了得到精 确的跃迁频率, 冷原子团所处的磁场需要尽可能地 补偿到零. 除了地磁场的贡献外, 离子泵所产生的 剩余磁场和补偿线圈电流精度都会对原子处的磁场 造成影响, 因此精确地标定原子处的磁场大小成为 抑制磁场的关键. 为了让各个速度分布的原子都参
与作用, 本实验采取同向拉曼共振的方法标定原子 处的磁场 ${ }^{[49]}$.

如图3所示, 将原子制备在 $2 \mathrm{~S}_{1 / 2}, \mathrm{~F}=1 / 2$ 基态上, 然后用单光子失谐为 $-2 \mathrm{GHz}$ 的同向拉曼光作用 在原子上. 通过改变拉曼光的双光子失谐, 探测 $2 \mathrm{~S}_{1 / 2}, \mathrm{~F}=3 / 2$ 的原子数, 可以得到拉曼共振谱. 通过 计算 ${ }^{6} \mathrm{Li}$ 原子在弱磁场下的塞曼能级䢃裂, 便可以得 到原子处的磁场大小. 调节补偿线圈的电流大小最

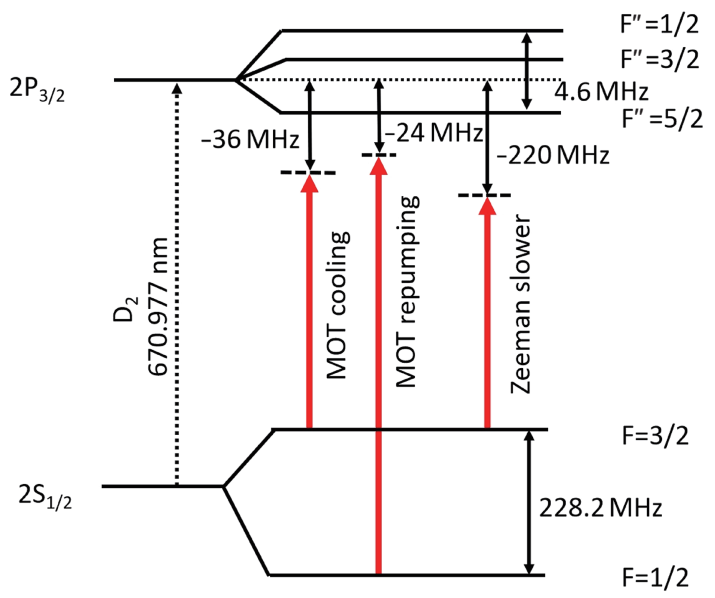

图 1 (网络版彩图) ${ }^{6} \mathrm{Li} \mathrm{MOT}$ 光能级图. 冷却光失谐 $-36 \mathrm{MHz}$, 再洜浦光失谐 $-24 \mathrm{MHz}$, 光斑大小为直径 $2 \mathrm{~cm}$

Figure 1 (Color online) The energy level diagram of ${ }^{6} \mathrm{Li}$ MOT. The frequency detunings of cooling and repumping laser light are -36 and $-24 \mathrm{MHz}$, respectively. The diameter of these laser beams is $2 \mathrm{~cm}$.

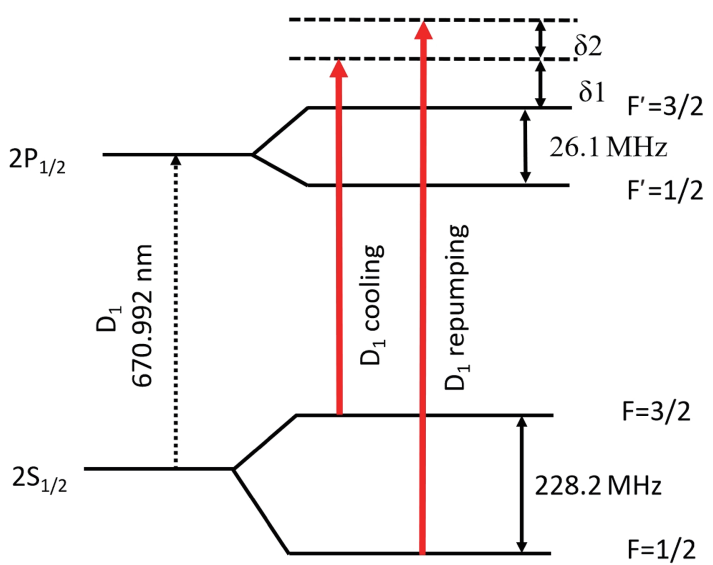

图 2 (网络版彩图) $\mathrm{D}_{1}$ 冷却光能级图. 冷却光和再泵浦光失 谐都为蓝失谐 $+30 \mathrm{MHz}$, 光斑大小为直径 $5.6 \mathrm{~mm}$

Figure 2 (Color online) The energy level diagram of ${ }^{6} \mathrm{Li}$ for $\mathrm{D}_{1}$ line gray cooling. The detunings of cooling beam and repumping beam are $+30 \mathrm{MHz}$ and the diameter of beam is $5.6 \mathrm{~mm}$. 


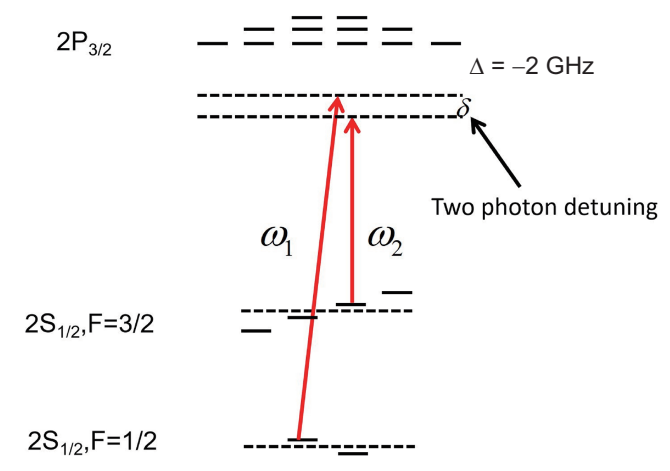

图 3 (网络版彩图)标定磁场的拉曼光能级图. $\omega_{1}$ 和 $\omega_{2}$ 为两 支同向拉曼光的频率, 单光子失谐为红失谐 $-2 \mathrm{GHz}$

Figure 3 (Color online) The configuration of Raman beams for calibrating background magnetic field. $\omega_{1}$ and $\omega_{2}$ are frequencies of Raman beams and the single photon detuning is $-2 \mathrm{GHz}$.

终将磁场控制在 8 mGauss 以下.

\section{3 光梳的稳定性传输}

如图4所示, 飞秒钛宝石激光器的超连续激 光的频率锁到窄线宽激光器上 ${ }^{[50]}$, 重复频率 $f_{r}=$ $998 \mathrm{MHz}$. 一方面, 窄线宽激光器锁定在超稳腔上, 超稳腔的秒稳优于 $1 \times 10^{-15}$. 但光梳的超稳腔随温 度变化而漂移, 会对频率测量带来影响. 在实验中, $f_{r}$ 每小时大约漂移 $2 \times 10^{-3} \mathrm{~Hz}$, 对应于光频的变化大 约为 $1 \mathrm{kHz}$. 每次测量时, 可实时记录 $f_{r}$, 将超稳腔带 来的频率漂移计入谱线测量的绝对频率中.

另一方面, 重频 $f_{r}$ 和零频 $f_{0}$ 都以氢钟作为参考, 积分时间大于 $1000 \mathrm{~s}$, 氢钟的频率稳定性为 $2 \times 10^{-15}$. 氢钟的参考频率通过全球定位系统(Global Positioning System, GPS)与美国海军天文台(United States Naval Observatory, USNO)的Cs原子钟相连. 通过长 时间对比氢钟和原子钟的 $1 \mathrm{pps}$ 信号, 发现两者的 时间间隔有微小的偏移. 如图5所示, 两条蓝线是 两次记录的信号, 红线是线性拟合, $\alpha 1$ 和 $\alpha 2$ 是线 性拟合的斜率, 表示的是氢钟和原子钟的频率偏 差. $\alpha 1=2.7858(5) \times 10^{-13}$ 意味着本地氢钟比原子钟

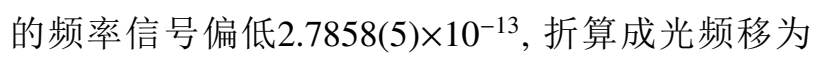
$134 \mathrm{~Hz}$. 同时, 两次测量氢钟的频率也在漂移, 漂移 量优于 $1 \times 10^{-13}$, 本实验取上限, 认为在测量时, 氢钟 的不确定度为 $1 \times 10^{-13}$, 对应的绝对频率的不确定度 为 $45 \mathrm{~Hz}$.
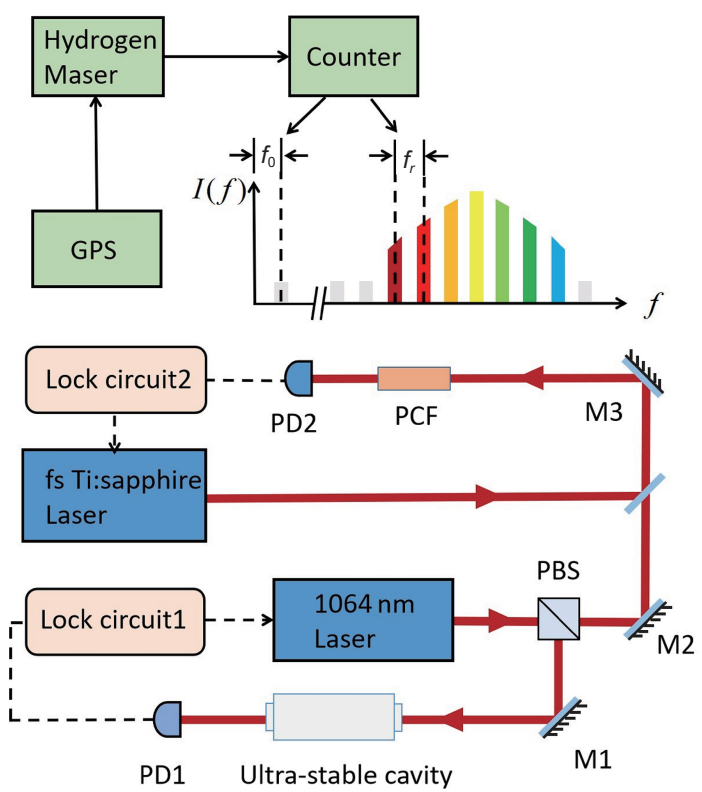

图 4 (网络版彩图)稳定性传递. 窄线宽 $1064 \mathrm{~nm}$ 激光器锁 定到超稳腔上, 飞秒钛宝石激光器锁定到窄线宽激光器上. PCF: 光子晶体光纤; PBS: 偏振极化棱镜; M: 全反镜; PD: 光 电探测器

Figure 4 (Color online) The stability transfer. The $1064 \mathrm{~nm}$ Laser with narrow linewidth is locked to ultra-stable cavity, and the fs Ti:sapphire is locked to $1064 \mathrm{~nm}$ laser. PCF: photonic crystal fiber; PBS: polarized beam splitter; M: mirror; PD: photodetector.

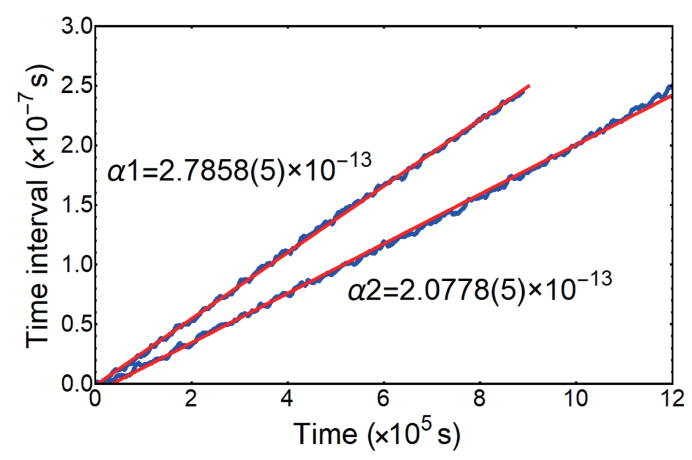

图 5 (网络版彩图)本地氢钟的稳定性. 蓝线为记录的数据, 红线为线性拟合, 斜率即为本地氢钟和原子钟的频率偏移

Figure 5 (Color online) The stability of local hydrogen maser. The blue dots are measured data and the red line is a linear fit. The slope of the linear fit represents the frequency shift between the maser and the atomic clock.

\section{4 光场散粒噪声极限的差拍探测}

为了最大程度地减少光洜浦和 $\mathrm{AC}$ stark效应, 实 验中探测光的功率被降低到几nW进行测量. 在探测 
光非常弱的情况下, 如果直接使用探测器采集光信 号, 信号将完全被噪声淹没. 相比于电放大的方法 可能带进来电子器件的噪声, 本实验使用差拍探测 的方法用强的本地光来放大探测光信号. 这样的光 放大可以仅将共振探测器共振频率处的信号进行放 大, 避免将其他频段的噪声放大.

由于使用的探测光是相干激光, 在一段时间内 的光子数会有起伏, 光子数的噪声即为散粒噪声. 理论上探测光的信噪比 ${ }^{[51]}$ 为 $\delta_{\text {shot }}=C \sqrt{\eta N_{\text {Probe }}}$, 其 中 $C$ 是模式匹配度, $\eta$ 是探测器量子效率, $N_{\text {Probe }}$ 是探 测光的光子数. 当探测信号的噪声大多数是由光子 散粒噪声贡献时, 认为此时达到光子的散粒噪声极 限. 在不同长度脉冲下, 可通过测量被本地光放大后 的探测光的噪声, 并与理论上光子的散粒噪声作比 较, 以此反映差拍探测是否达到散粒噪声极限.

在差拍探测中, 探测光和本地光之间的模式匹 配非常关键, 越接近 $100 \%$ 越容易达到散粒噪声极 限. 在本实验的测量中, 两束光的拍频干涉对比度优 于 $97 \%$. 差拍探测的噪声测量如图6所示, 将弱探测 光的噪声归一化为理论的散粒噪声. 对于 $1-1000 \mu \mathrm{s}$ 的脉冲时间, 归一化的噪声非常接近 1 , 即达到光 子散粒噪声极限. 脉冲宽度小于 $1 \mu \mathrm{s}$ 时, 对应的归 一化噪声小于 1 是由测量系统中的低通滤波器造成 的. 脉冲宽度大于 $1000 \mu \mathrm{s}$ 时, 对应的归一化噪声大 于1是由测量时探测光光强的缓慢变化和相对相位 的漂移导致.

在实验中, 垂直线偏的两束探测光对打探测冷 原子, 再用携带原子信息的探测光和本地光拍频, 分 别用两个共振频率为 $21 \mathrm{MHz}$ 的共振探测器接收信 号. 然后分别将共振探测器的电信号和相位差 $90^{\circ}$ 的 $21 \mathrm{MHz}$ 电信号进行混频. 两个混频器得到的混频 信号 $S_{I}$ 和 $S_{Q}$ 分别平方再相加, 就能够得到原子吸收 谱线信号 $S_{T}$, 所发展的这种探测手段可以有效解决 在普通差拍探测中对探测光的相对相位敏感问题.

\section{5 实验光路}

实验装置示意图如图7所示. 首先制得原子 数为 $5 \times 10^{7}$, 温度为 $50 \mu \mathrm{K}$ 的冷原子团. 再用一个输 出 $30 \mathrm{~mW}$ 的外腔反馈半导体激光器提供本地光和探
测光. 同时分出一路光和光梳拍频, 将激光器锁到 光梳上. AOM1用来扫频及锁定功率, 通过光强锁定 将功率起伏抑制在 $1 \%$ 以下. 用差拍探测的方法测量 原子的吸收谱. 探测光和本地光的光频差为 $21 \mathrm{MHz}$, 由 AOM2和AOM3控制. 探测光在原子团处的光斑 直径约为 $2.6 \mathrm{~mm}$, 两束探测光角度小于 $200 \mu \mathrm{rad}$, 光 强不平衡度小于 $5 \times 10^{-3}$. 探测光光强在 $\mathrm{nW}$ 量级, 本

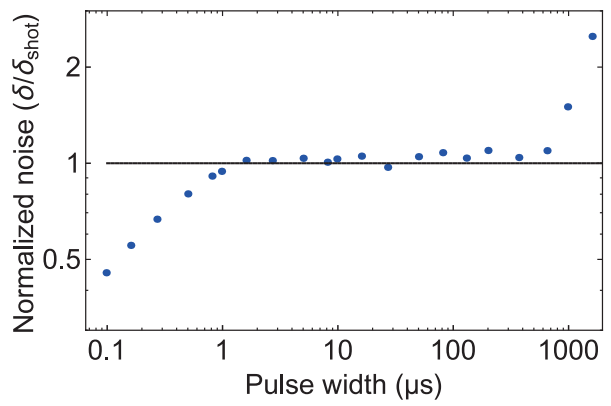

图 6 (网络版彩图)差拍探测的散粒噪声测量. 本地光为 $600 \mu \mathrm{W}$, 探测光为 $30 \mathrm{nW}$, 持续时间为 $0.1-1000 \mu \mathrm{s}$

Figure 6 (Color online) Shot noise of heterodyne detection. The power of local beam and probe beam are $600 \mu \mathrm{W}$ and $30 \mathrm{nW}$ and the duration time from 0.1 to $1000 \mu \mathrm{s}$.

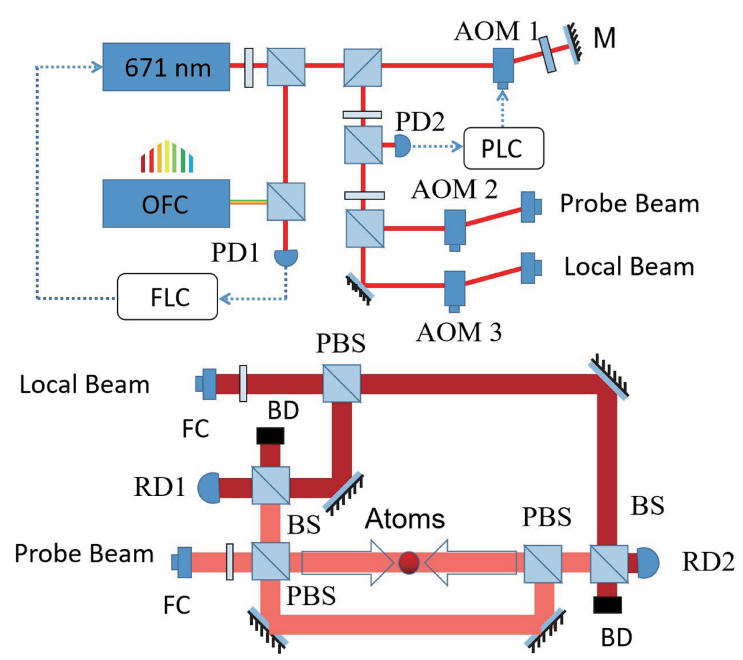

图 7 (网络版彩图)实验光路图. PBS: 偏振极化棱镜; BS: 极 化棱镜; $\mathrm{M}$ : 全反镜; $\mathrm{AOM}$ : 声光调制器; $\mathrm{PD}$ : 光电探测器; $\mathrm{RD}$ : 共振探测器; BD: 光束收集器; FC: 光纤耦合头; FLC: 频率锁 定电路; PLC: 功率锁定电路

Figure 7 (Color online) Setup of the experiment. PBS: polarized beam splitter; BS: beam splitter; M: mirror; AOM: acousto-optic modulator; PD: photodetector; RD: resonance detector; BD: beam collcetor; FC: fiber coupler; FLC: frequency locking circuit; PLC: power locking circuit. 
地光约 $600 \mu \mathrm{W}$. 探测光和本地光通过 BS进行拍频, 用透镜将光汇聚到共振探测器靶面上.

\section{3 实验结果}

\section{1 原子跃迁绝对频率}

在频率测量时, 弱探测光的脉冲持续时间固定 为 $200 \mu \mathrm{s}$, 在这段时间内扫描探测光的频率, 即可 获得原子跃迁的吸收谱线. 如图8所示, 跃迁为 $2 \mathrm{~S}_{1 / 2}$, $\mathrm{F}=3 / 2 \rightarrow 2 \mathrm{P}_{1 / 2}, \mathrm{~F}^{\prime}=3 / 2$ 的吸收光谱及其对应的拟合曲 线. 拟合线宽约为 $7.37 \mathrm{MHz}$, 比 $5.8 \mathrm{MHz}$ 的自然线宽 大 $25 \%$, 这主要是由残留的多普勒效应导致.

随着探测光光功率密度的减小, 绝对频率的 测量值会发生偏移. 图9给出了 $2 \mathrm{~S}_{1 / 2}, \mathrm{~F}=3 / 2 \rightarrow 2 \mathrm{P}_{1 / 2}$, $F^{\prime}=3 / 2$ 跃迁的绝对频率与探测光光功率密度变化 的关系, 其中虚线表示NIST 在2011年测量 ${ }^{[42]}$ 的参 考频率 $f_{\text {rel }}\left(f_{\text {rel }}=446789528.716(10) \mathrm{MHz}\right)$. 显然, 由 探测光光强引起的频移在 $\mathrm{kHz}$ 量级, 是不可忽略的. 当光强从 $1 \mu \mathrm{W} / \mathrm{cm}^{2}$ 增强为 $8 \mu \mathrm{W} / \mathrm{cm}^{2}$ 时, 可观察到大 约 $30 \mathrm{kHz}$ 的偏移. 在 $6 \mu \mathrm{W} / \mathrm{cm}^{2}$ 的条件下, 本实验获 得了和NIST相接近的值. 最终的绝对频率是将测量 值通过线性拟合把光强外推至零处而获得的.

表1给出了实验测量的所有能级跃迁的频率以 及 ${ }^{6} \mathrm{Li}$ 的 $\mathrm{D}_{1}$ 和 $\mathrm{D}_{2}$ 线的中心频率.

\section{2 不确定度分析}

如表2所示, 考虑每个误差来源所带来的不确

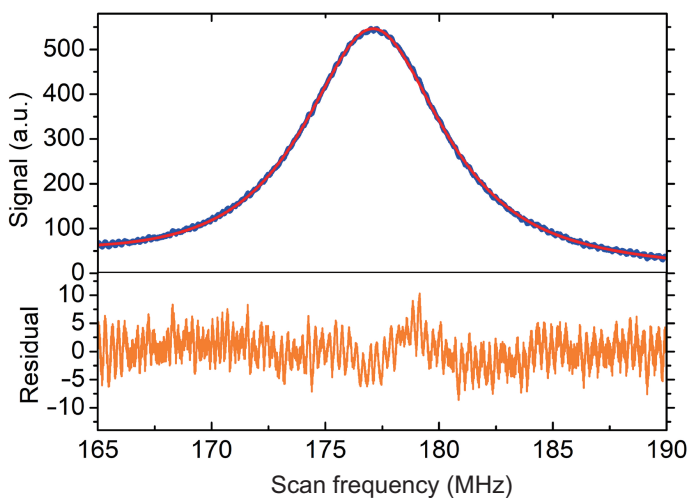

图 8 (网络版彩图) ${ }^{6} \mathrm{Li} \mathrm{D}_{1}$ 线吸收信号拟合和残差. 上图红线 是拟合图形,下图橙线为拟合残差

Figure 8 (Color online) The absorbtion signal of $\mathrm{D}_{1}$ transition of ${ }^{6} \mathrm{Li}$. Red line is fitting curve and orange line is fitting residual.

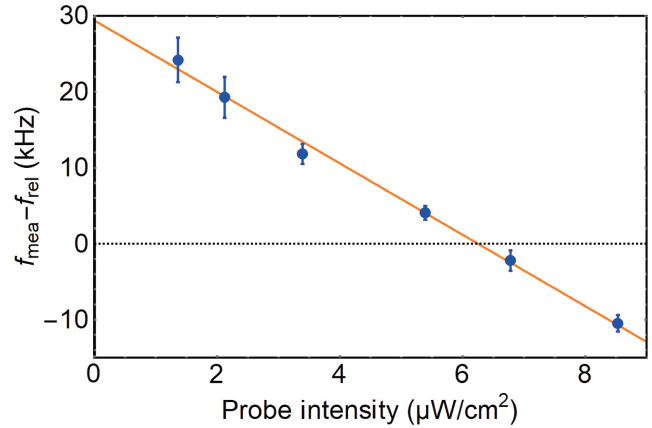

图 9 (网络版彩图)随着探测光光强变化, 频率测量会有一 定的偏移. 蓝点是测量数据, 橙线是线性拟合

Figure 9 (Color online) The measured frequency shift as a function of the probe beam intensity. Blue dots are the measured data and orange solid line is a linear fit to extract the frequency with zero intensity of probe beams.

表 $1{ }^{6} \mathrm{Li}$ 跃迁频率和 $\mathrm{D}_{1}$ 线 $\mathrm{D}_{2}$ 线的中心频率

Table 1 Measured frequencies of hyperfine components and centers of gravity (COG) of the ${ }^{6} \mathrm{Li} \mathrm{D}$ lines

\begin{tabular}{cccc}
\hline 跃迁 & 基态 $\mathrm{F}$ & 激发态 $\mathrm{F}$ & 频率 $(\mathrm{MHz})$ \\
\hline & $3 / 2$ & $1 / 2$ & $446789502.6393(9)$ \\
$2 \mathrm{~S}_{1 / 2} \rightarrow 2 \mathrm{P}_{1 / 2}$ & $3 / 2$ & $3 / 2$ & $446789528.7442(9)$ \\
& $1 / 2$ & $1 / 2$ & $446789730.8424(23)$ \\
& $1 / 2$ & $3 / 2$ & $446789756.9439(8)$ \\
\hline $\mathrm{D}_{1} \mathrm{COG}$ & & & $446789596.1094(8)$ \\
\hline & $3 / 2$ & $5 / 2$ & $446799571.0796(19)$ \\
$2 \mathrm{~S}_{1 / 2} \rightarrow 2 \mathrm{P}_{3 / 2}$ & $3 / 2$ & $3 / 2$ & $446799573.9743(19)$ \\
& $1 / 2$ & $1 / 2$ & $446799575.6861(19)$ \\
& $1 / 2$ & $3 / 2$ & $446799802.1758(16)$ \\
\hline $\mathrm{D}_{2} \mathrm{COG}$ & & & $446799803.8876(16)$ \\
\hline
\end{tabular}

表 2 绝对频率的不确定度组成

Table 2 Uncertainty budgets (Hz) for the measurement

\begin{tabular}{ccc}
\hline 不确定度 & $\mathrm{D}_{1}$ 线 $(\mathrm{Hz})$ & $\mathrm{D}_{2}$ 线 $(\mathrm{Hz})$ \\
\hline 统计误差 & 813 & 1048 \\
光梳参考误差 & 45 & 45 \\
一阶多普勒效应 & 375 & 375 \\
磁场 & 57 & 57 \\
超精细结构常数 & 0 & 1150 \\
局域场效应 & 3 & 6 \\
AC Stark效应 & 40 & 10 \\
碰撞 & $<100$ & $<100$ \\
\hline 合计 & 906 & 1606
\end{tabular}


定度, 总不确定度是将各个不确定度矢量叠加得到. 对于可分辨的 $\mathrm{D}_{1}$ 线, 测量不确定度小于 $1 \mathrm{kHz}$, 对于 不可分辨的 $\mathrm{D}_{2}$ 线, 其不确定度小于 $2 \mathrm{kHz}$.

\subsection{1一阶多普勒效应}

原子团温度为 $50 \mu \mathrm{K}$, 其最概然速度是 $0.37 \mathrm{~m} / \mathrm{s}$, 对应的一阶多普勒展宽为 $551.2 \mathrm{kHz}$. 通过对打探测 光的方式对测量的绝对频率进行抑制, 精细调节两 支探测光的角度, 使角度差在 $200 \mu \mathrm{rad}$ 以下, 对应的 不确定度约为 $110 \mathrm{~Hz}$.

此外, 由于锂原子较轻, 需要考虑探测光对原子 的推动. 受限于半波片和PBS的控制精度, 两支探测 光的光强不平衡度优于 $5 \%$, 这将带来 $360 \mathrm{~Hz}$ 的不确 定度. 总的多普勒效应将带来 $375 \mathrm{~Hz}$ 的不确定度.

\subsection{2 磁场}

在弱磁场情况下, 磁子能级会发生能级偏移, 对 于不同探测光的偏振, 会导致原子的跃迁频率发生 偏移. 但同时, 总有与之对应的一种偏振将这一偏 移抵消, 使得跃迁频率的测量值产生展宽. 然而不 同磁子能级的原子数会有很小的差异, 这一差异使 得这一对跃迁的谱线强度不一致, 最终导致跃迁频 率有微小的偏移.

在本实验中, 原子布居数不平衡在 $0.7 \%$, 磁子 能级之间的最大间隔为 $28 \mathrm{kHz}$, 通过拟合得到因磁 场带来的偏移为 $57 \mathrm{~Hz}$. 由于不确定剩余磁场方向, 磁场带来的噪声取上限 $57 \mathrm{~Hz}$.

\subsection{3 超精细结构常数}

由于 $\mathrm{D}_{2}$ 线的䢃裂只有 $4.6 \mathrm{MHz}$, 小于自然线宽, 不可分辨, 本实验采取固定能级间隔的方法拟 合 $\mathrm{D}_{2}$ 线的绝对频率. 此时, 造成 $\mathrm{D}_{2}$ 线频率的不确定 性的最大因素是超精细结构常数 ${ }^{[9]}$ 的不准确.

图10所示为 ${ }^{6} \mathrm{Li} 2 \mathrm{P}_{3 / 2}$ 的超精细结构能级. 每个 超精细结构能级的偏移量:

$\Delta E_{H F}=\frac{1}{2} A C+\frac{3}{8} B \frac{C(C+1)}{I(2 I-1) J(2 J-1)}$,

其中 $C=F(F+1)-J(J+1)-I(I+1) . A$ 和 $B$ 分别是 磁偶级超精细常数和电四极超精细常数, 能级间隔 splitting $1=1.7118(12) \mathrm{MHz}$, splitting2=2.8947(7) MHz. 在对吸收谱线进行拟合时, 需要考虑能级间隔的 误差对拟合结果带来的不确定度, 本文认为, 这 个不确定度近似于splitting1和splitting2的不确定度, 即f5, f6, f7的不确定度为 $1350 \mathrm{~Hz}, \mathrm{f} 8, \mathrm{f} 9$ 的不确定度 为 $1150 \mathrm{~Hz}$.

\subsection{4 局域场效应}

由于测量的是吸收谱, 需要对局域场效应做 出估算. 由于实验中较小的原子数密度 $\left(n_{0} k^{-3}=\right.$ $\left.3.8 \times 10^{-6}, k=2 \pi / \lambda\right)$ 和弱探测光, 来自散射的集体 效应也很小 ${ }^{[52]}$. 在低阶近似下, 当探测光频率扫过 共振时, 会存在Lorentz shift ${ }^{[53]}$ :

$\Delta L L=\frac{-n_{0} d^{2}}{3 \varepsilon_{0} \hbar}$,

其中 $n_{0}$ 是原子数密度, $d$ 是跃迁偶极矩, $\varepsilon_{0}$ 是真空介 电常数. 由局域场效应引起的频移约为 $\mathrm{D}_{1}$ 线 $-28 \mathrm{~Hz}$, $\mathrm{D}_{2}$ 线 $-57 \mathrm{~Hz}$. 考虑原子数密度的抖动和探测光的抖 动, 取绝对频率的不确定度为 $3 \mathrm{~Hz}$ 和 $6 \mathrm{~Hz}$.

\subsection{5 量子干涉}

在 $\mathrm{D}_{2}$ 跃迁谱线的测量中, 与NIST的测量类 似 ${ }^{[42]}$, 由于 $\mathrm{D}_{2}$ 跃迁谱线间隔太近, 需要考虑量子 干涉带来的影响 ${ }^{[43]}$.

以 $2 \mathrm{~S}_{1 / 2}, \mathrm{~F}=1 / 2 \rightarrow 2 \mathrm{P}_{3 / 2}$ 跃迁为例, 加入了量子干 涉修正后的谱线拟合如图11所示, 本实验通过固定 两个跃迁峰的间隔进行拟合, 两者之间的频率间 隔 $1.712 \mathrm{MHz}$. 从图中可以看出, 量子干涉部分对于 整个谱线影响较大, 所以不同能级之间的量子干涉 不可忽略.

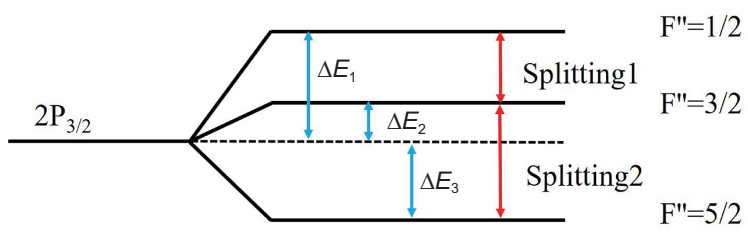

图 10 (网络版彩图) ${ }^{6} \mathrm{Li}$ 的 $2 \mathrm{P}_{3 / 2}$ 能级超精细结构䢃裂

Figure 10 (Color online) $2 \mathrm{P}_{3 / 2}$ hyperfine structure energy levels of ${ }^{6} \mathrm{Li}$. 


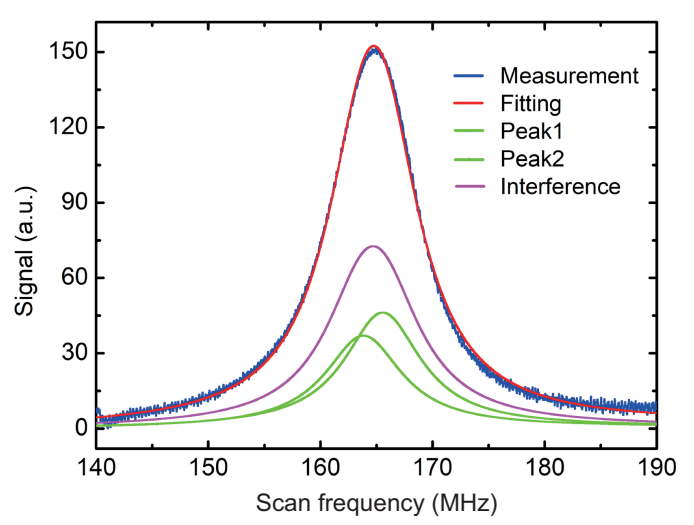

图 11 (网络版彩图) $2 \mathrm{~S}_{1 / 2}, \mathrm{~F}=1 / 2 \rightarrow 2 \mathrm{P}_{3 / 2}$ 跃迁有苂光干涉修 正拟合

Figure 11 (Color online) Fitting with quantum interference correction for $2 \mathrm{~S}_{1 / 2}, \mathrm{~F}=1 / 2 \rightarrow 2 \mathrm{P}_{3 / 2}$ transition.

\subsection{6 其他误差}

系统的其他不确定性, 例如光洜浦、多激发光 子反冲和AC Stark效应, 由于探测光非常弱, 并且本 实验通过光强外推获得的绝对频率, 因此不确定度 很小.

应当指出, $2 \mathrm{~S}_{1 / 2}, \mathrm{~F}=1 / 2 \rightarrow 2 \mathrm{P}_{1 / 2}, \mathrm{~F}^{\prime}=3 / 2$ 的跃迁 概率是 $2 \mathrm{~S}_{1 / 2}, \mathrm{~F}=1 / 2 \rightarrow 2 \mathrm{P}_{1 / 2}, \mathrm{~F}^{\prime}=1 / 2$ 的 8 倍. 为了获得 最佳的信噪比, 本实验在测量 $2 \mathrm{~S}_{1 / 2}, \mathrm{~F}=1 / 2 \rightarrow 2 \mathrm{P}_{1 / 2}$, $F^{\prime}=1 / 2$ 这一跃迁时, 增加了原子团的光学密度.

\section{3 对比分析}

从 20 世纪 50 年代开始, 就有人开始测量 ${ }^{6} \mathrm{Li}$ 的精 细结构能级 ${ }^{[41,54-56]}$, 随着测量方法的不断改进, 测 量的精度也在不断提高. 与此同时, 由于 ${ }^{6} \mathrm{Li}$ 外层只 有三个电子, 便于理论计算, 理论模型也在不断修 正. 图12所示为几十年来实验测量和理论计算的精 细结构䢃裂的不确定度.

目前Das和Sansonetti等人 ${ }^{[36,42]}$ 报道了 ${ }^{6} \mathrm{Li}$ 的绝 对跃迁频率的精确测量结果. 本实验的测量结果 和Das的相比, D线跃迁频率值差了 $20-85 \sigma$. 本实验 的测量结果和NIST的测量值相比, 测量频率的不 确定度小一个数量级, $\mathrm{D}_{1}$ 线的绝对跃迁频率偏高2$28 \mathrm{kHz}$. 本文认为, 这些差异可能是由于测量中大光 强引起的频率偏移. 对于 $\mathrm{D}_{2}$ 线, 本实验的结果与他 们的结果在 $1 \sigma$ 量级上是一致的.

本实验的精细结构䢃裂和超精细结构䢃裂结
果与先前实验的比较如表3所示. 测得的 $2 \mathrm{P}_{1 / 2}$ 超 精细结构䢃裂为26.1031(14) MHz, 这与最近的理 论计算26.1026(6) MHz非常吻合 ${ }^{[8]}$. 本实验测量基 态超精细结构䢃裂的结果是228.2015(14) MHz, 与 Beckmann等人 ${ }^{[37]}$ 用原子束磁共振方法测量的结果 相差 $2.6 \sigma$. 他们测量的不确定度号称为 $\mathrm{Hz}$, 比本实验 的测量精度高约 3 个量级. 在这个量级中, 必须考虑 更多的系统误差, 需要进一步研究两者之间的差异.

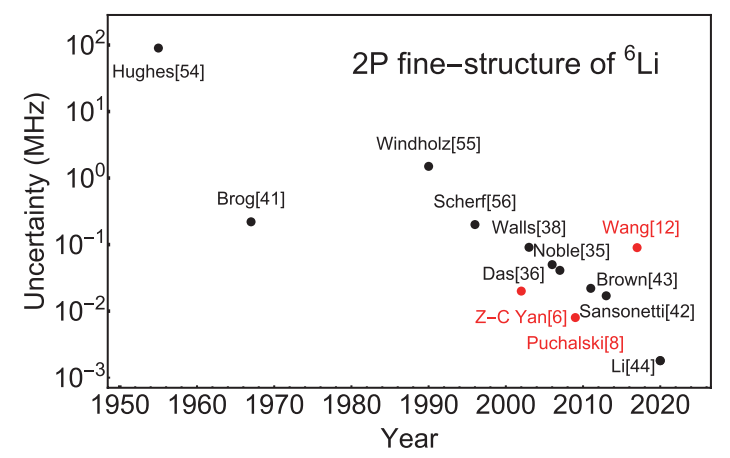

图 12 (网络版彩图)不同小组实验或计算得到的 ${ }^{6} \mathrm{Li} 2 \mathrm{P}$ 精细 结构能级的不确定度

Figure 12 (Color online) Uncertainty of $2 \mathrm{P}$ fine-structure of ${ }^{6} \mathrm{Li}$ from different groups.

表 3 不同理论计算和实验测量的 ${ }^{6} \mathrm{Li}$ 超精细结构能级幦裂 和精细结构能级䢃裂

Table 3 Fine structure splitting and hyperfine structure splitting of ${ }^{6} \mathrm{Li}$ from different groups

\begin{tabular}{|c|c|c|c|}
\hline 时间间隔 & 能级䢃裂 (MHz) & 参考文献 & 年份 \\
\hline \multirow{3}{*}{$2 \mathrm{~S} \mathrm{hfs}$} & $228.215(17)$ & Sansonetti等人 [42] & 2011 \\
\hline & $228.205261(3)$ & Beckmann等人 [37] & 1974 \\
\hline & $228.2015(14)$ & $\mathrm{Li}$ 等人 [44] & 2020 \\
\hline \multirow{7}{*}{$2 \mathrm{P}_{1 / 2} \mathrm{hfs}$} & $26.111(15)$ & Sansonetti等人 [42] & 2011 \\
\hline & $26.091(6)$ & Das等人 [36] & 2007 \\
\hline & $26.079(46)$ & Walls等人 [38] & 2003 \\
\hline & $26.110(56)$ & Noble等人 [35] & 2006 \\
\hline & $26.1026(6)$ & Puchalski等人 $[8]$ & 2009 \\
\hline & $26.1087(8)$ & Yerokhin $^{[9]}$ & 2008 \\
\hline & $26.1031(14)$ & $\mathrm{Li}$ 等人 ${ }^{[44]}$ & 2020 \\
\hline \multirow{7}{*}{$2 \mathrm{P}$ fs } & 10052.72(6) & Puchalski等人[11] & 2014 \\
\hline & $10052.799(22)$ & Sansonetti等人 [42] & 2011 \\
\hline & $10052.76(22)$ & Brog等人 [41] & 1967 \\
\hline & $10052.964(50)$ & Noble等人 [35] & 2006 \\
\hline & $10052.044(91)$ & Walls等人 [38] & 2003 \\
\hline & $10052.862(41)$ & Das等人 [36] & 2007 \\
\hline & $10052.7804(18)$ & Li等人 [44] & 2020 \\
\hline
\end{tabular}


${ }^{6} \mathrm{Li}$ 的 $2 \mathrm{P}$ 能 级 精 细结构 䢃 裂 的测 量 值为 10052.7804(18) MHz, 这与最新的测量结果 ${ }^{[42] 一}$ 一致, 与 $\mathrm{m} \alpha^{6}$ 和 $\mathrm{m} \alpha^{7} \operatorname{In} \alpha$ 修正的理论 ${ }^{[11,12]}$ 也是一致的.

\section{4 结论}

本实验实现了光子散粒噪声极限的 ${ }^{6} \mathrm{Li}$ 精密光
谱, 使用光学频率梳测量了 ${ }^{6} \mathrm{Li}$ 冷原子的D线跃迁的 绝对频率. 测量精度比目前最好的实验结果提高了 一个数量级. 这些测量结果与最近由高阶相对论 和QED修正的2P精细结构䢃裂计算结果符合得很 好, 并有助于解决理论与实验之间的巨大分歧. 若将 此类测量推广到 ${ }^{6} \mathrm{Li}$ 和 ${ }^{7} \mathrm{Li}$ 的冷原子, 将在更高精度 的水平上确定同位素位移和相对核电荷半径.

\section{参考文献}

1 Yan Z C, Drake G W F. Lithium fine structure in the $1 \mathrm{~s}^{2} 2 \mathrm{p}^{2} \mathrm{P}_{J}$ states. Phys Rev Lett, 1997, 79: 1646-1649

2 Guan X X, Wang Z W. The hyperfine structure of the $1 \mathrm{~s}^{2} n \mathrm{~s}^{2} \mathrm{~S}$ and $1 \mathrm{~s}^{2} n \mathrm{p}^{2} \mathrm{P}$ states $(n=2,3,4$, and 5) for the lithium isoelectronic sequence. Eur Phys J D, 1998, 2: 21-27

3 Yamanaka N. Multiconfigurational Hartree-Fock calculations of the hyperfine structure constants for the $1 \mathrm{~s}^{2} 2 \mathrm{~s}^{2} \mathrm{~S}$ and $1 \mathrm{~s}^{2} 2 \mathrm{p}^{2} \mathrm{P}$ states of ${ }^{7} \mathrm{Li}$ and ${ }^{9} \mathrm{Be}^{+}$. J Phys Soc Jpn, 1999, 68: 2561-2569

4 Yan Z C, Drake G W F. Lithium isotope shifts as a measure of nuclear size. Phys Rev A, 2000, 61: 022504

5 Godefroid M, Fischer C F, Jönsson P. Non-relativistic variational calculations of atomic properties in Li-like ions: Li I to O VI. J Phys B, 2001, 34: 1079-1104

6 Yan Z C, Drake G W F. Lithium transition energies and isotope shifts: QED recoil corrections. Phys Rev A, 2002, 66: 042504

7 Yan Z C, Nörtershäuser W, Drake G W F. High precision atomic theory for Li and Be+ : QED shifts and isotope shifts. Phys Rev Lett, 2008, 100: 243002

8 Puchalski M, Pachucki K. Fine and hyperfine splitting of the 2P state in $\mathrm{Li}$ and Be ${ }^{+}$. Phys Rev A, 2009, 79: 032510

9 Yerokhin V A. Hyperfine structure of $\mathrm{Li}$ and $\mathrm{Be}^{+}$. Phys Rev A, 2008, 78: 012513

10 Puchalski M, Moro A M, Pachucki K. Isotope shift of the $3^{2} S_{1 / 2}-2^{2} S_{1 / 2}$ transition in lithium and the nuclear polarizability. Phys Rev Lett, 2006, 97: 133001

11 Puchalski M, Pachucki K. Quantum electrodynamics corrections to the 2P fine splitting in Li. Phys Rev Lett, 2014, 113: 073004

12 Wang L M, Li C, Yan Z C, et al. Isotope shifts and transition frequencies for the S and P states of lithium: Bethe logarithms and second-order relativistic recoil. Phys Rev A, 2017, 95: 032504

13 Minardi F, Bianchini G, Pastor P C, et al. Measurement of the helium $2^{3} \mathrm{P}_{0}-2^{3} \mathrm{P}_{1}$ fine structure interval. Phys Rev Lett, 1999, 82: 1112-1115

14 Zelevinsky T, Farkas D, Gabrielse G. Precision measurement of the three $2^{3} \mathrm{P}_{J}$ helium fine structure intervals. Phys Rev Lett, 2005, $95: 203001$

15 Borbely J S, George M C, Lombardi L D, et al. Separated oscillatory-field microwave measurement of the $2^{3} \mathrm{P}_{1}-2^{3} \mathrm{P}_{2}$ fine-structure interval of atomic helium. Phys Rev A, 2009, 79: 060503

16 Smiciklas M, Shiner D. Determination of the fine structure constant using helium fine structure. Phys Rev Lett, 2010, 105: 123001

17 Zheng X, Sun Y R, Chen J J, et al. Laser spectroscopy of the fine-structure splitting in the $2^{3} \mathrm{P}_{J}$ levels of ${ }^{4} \mathrm{He}$. Phys Rev Lett, 2017, $118: 063001$

18 Zheng X, Sun Y, Chen J J, et al. Precision spectroscopy on the $2^{3}$ S $-2^{3} \mathrm{P}$ transition of atomic helium. Acta Phys Sin, 2018, 67: 164203

19 Johnson W R, Safronova U I, Derevianko A, et al. Relativistic many-body calculation of energies, lifetimes, hyperfine constants, and polarizabilities in ${ }^{7}$ Li. Phys Rev A, 2008, 77: 022510

20 Derevianko A, Porsev S G, Beloy K. Convergence of all-order many-body methods: Coupled-cluster study for Li. Phys Rev A, 2008, 78: 010503

21 Bieron $\mathrm{J}$, Jönsson P, Froese Fischer C. Effects of electron correlation, relativity, and nuclear structure on hyperfine constants of Be ${ }^{+}$and $\mathrm{F}^{6+}$. Phys Rev A, 1999, 60: 3547-3557

22 Shiner D, Dixson R, Vedantham V. Three-nucleon charge radius: A precise laser determination using ${ }^{3}$ He. Phys Rev Lett, 1995, 74: $3553-3556$

23 De Jager C W, De Vries H, De Vries C. Nuclear charge- and magnetization-density-distribution parameters from elastic electron scattering. Atomic Data Nucl Data Tables, 1974, 14: 479-508

24 Drake G. Long-range casimir forces: Theory and recent experiments on atomic systems. Phys Today, 1993, 47: 7, 67

25 Marin F, Minardi F, Pavone F S, et al. Hyperfine structure of the $3^{3} \mathrm{P}$ state of ${ }^{3} \mathrm{He}$ and isotope shift for the $2^{3} \mathrm{~S}-3^{3} \mathrm{P}_{0}$ transition. Z Phys D, 1995, 32: 285-293

26 Riis E, Sinclair A G, Poulsen O, et al. Lamb shifts and hyperfine structure in ${ }^{6} \mathrm{Li}^{+}$and ${ }^{7} \mathrm{Li}^{+}$: Theory and experiment. Phys Rev A, 1994, 49: 
207-220

27 Tanihata I, Hamagaki H, Hashimoto O, et al. Measurements of interaction cross sections and nuclear radii in the light p-shell region. Phys Rev Lett, 1985, 55: 2676-2679

28 Ewald G, Nörtershäuser W, Dax A, et al. Nuclear charge radii of ${ }^{8,9}$ Li determined by laser spectroscopy. Phys Rev Lett, 2004, $93: 113002$

29 Sánchez R, Nörtershäuser W, Ewald G, et al. Nuclear charge radii of ${ }^{9,11} \mathrm{Li}$ : The influence of halo neutrons. Phys Rev Lett, 2006, $96: 033002$

30 Jensen A S, Riisager K, Fedorov D V, et al. Structure and reactions of quantum halos. Rev Mod Phys, 2004, 76: 215-261

31 Tanihata I. Neutron halo nuclei. J Phys G-Nucl Part Phys, 1996, 22: 157-198

32 Wang L B, Mueller P, Bailey K, et al. Laser spectroscopic determination of the ${ }^{6} \mathrm{He}$ nuclear charge radius. Phys Rev Lett, 2004, 93 : 142501

33 Pieper S C, Wiringa R B. Quantum Monte Carlo calculations of light nuclei. Annu Rev Nucl Part Sci, 2001, 51: 53-90

34 Bushaw B A, Nörtershäuser W, Ewald G, et al. Hyperfine splitting, isotope shift, and level energy of the 3S states of ${ }^{6,7} \mathrm{Li}$. Phys Rev Lett, 2003, 91: 043004

35 Noble G A, Schultz B E, Ming H, et al. Isotope shifts and fine structures of ${ }^{6,7} \mathrm{Li} \mathrm{D}$ lines and determination of the relative nuclear charge radius. Phys Rev A, 2006, 74: 012502

36 Das D, Natarajan V. Absolute frequency measurement of the lithium D lines: Precise determination of isotope shifts and fine-structure intervals. Phys Rev A, 2007, 75: 052508

37 Beckmann A, Böklen K D, Elke D. Precision measurements of the nuclear magnetic dipole moments of ${ }^{6} \mathrm{Li},{ }^{7} \mathrm{Li},{ }^{23} \mathrm{Na},{ }^{39} \mathrm{~K}$ and ${ }^{41} \mathrm{~K}$. Z Physik, 1974, 270: 173-186

38 Walls J, Ashby R, Clarke J J, et al. Measurement of isotope shifts, fine and hyperfine structure splittings of the lithium D lines. Eur Phys J D, 2003, 22: $159-162$

39 Huang Y C, Luo W J, Kuo Y T, et al. Precision measurement of hyperfine intervals in the $\mathrm{D}_{1}$ lines of atomic ${ }^{7}$ Li. J Phys B At Mol Opt Phys, 2013, 46: 075004

40 Lien Y H, Lo K J, Chen H C, et al. Absolute frequencies of the ${ }^{6,7} \mathrm{Li} 2 \mathrm{~S}^{2} S_{1 / 2} \rightarrow 3 \mathrm{~S}^{2} S_{1 / 2}$ transitions. Phys Rev A, 2011, 84: 042511

41 Brog K C, Eck T G, Wieder H. Fine and hyperfine structure of the $2{ }^{2} \mathrm{P}$ term of ${ }^{6} \mathrm{Li}$ and ${ }^{7} \mathrm{Li}$. Phys Rev, 1967, 153: 91-103

42 Sansonetti C J, Simien C E, Gillaspy J D, et al. Absolute transition frequencies and quantum interference in a frequency comb based measurement of the ${ }^{6,7} \mathrm{Li}$ D lines. Phys Rev Lett, 2011, 107: 023001

43 Brown R C, Wu S, Porto J V, et al. Quantum interference and light polarization effects in unresolvable atomic lines: Application to a precise measurement of the ${ }^{6,7} \mathrm{Li} \mathrm{D}_{2}$ lines. Phys Rev A, 2013, 87: 032504

44 Li R, Wu Y, Rui Y, et al. Absolute frequency measurement of ${ }^{6} \mathrm{Li}$ D lines with kHz-level uncertainty. Phys Rev Lett, 2020, 124: 063002

45 Deng S, Chenu A, Diao P, et al. Superadiabatic quantum friction suppression in finite-time thermodynamics. Sci Adv, 2018, 4: eaar5909

46 Deng S, Shi Z Y, Diao P, et al. Observation of the Efimovian expansion in scale-invariant Fermi gases. Science, 2016, 353: 371-374

47 Grier A T, Ferrier-Barbut I, Rem B S, et al. Enhanced sub-Doppler cooling of lithium atoms in D 1 gray molasses. Phys Rev A, 2013, 87: 063411

48 Wu Y L, Li R, Rui Y, et al. Precise measurement of ${ }^{6} \mathrm{Li}$ transition frequencies and hyperfine splitting. Acta Phys Sin, 2018, 67: 163201

49 Savard T A, Granade S R, O'Hara K M, et al. Raman-induced magnetic resonance imaging of atoms in a magneto-optical trap. Phys Rev A, 1999, 60: $4788-4795$

50 Chen H Q, Jiang Y Y, Bi Z Y, et al. Progress and trend of narrow-linewidth lasers. Sci China Technol Sci, 2013, 56: 1589-1596

51 Pino J M, Wild R J, Makotyn P, et al. Photon counting for Bragg spectroscopy of quantum gases. Phys Rev A, 2011, 83: 033615

52 Zhu B, Cooper J, Ye J, et al. Light scattering from dense cold atomic media. Phys Rev A, 2016, 94: 023612

53 Lorentz H. The Theory of Electrons. Leipzig: Teubner, 1916

54 Hughes R H. Isotope shift in the first spectrum of atomic lithium. Phys Rev, 1955, 99: 1837-1839

55 Windholz L, Jäger H, Musso M, et al. Laserspectroscopic investigations of the lithium-D-lines in magnetic fields. Z Phys D, 1990, 16: 41-47

56 Scherf W, Khait $\mathrm{O}$, Jäger H, et al. Re-measurement of the transition frequencies, fine structure splitting and isotope shift of the resonance lines of lithium, sodium and potassium. Z Phys D, 1996, 36: 31-33 


\title{
Precise absolute frequency measurement of three-electron ${ }^{6} \mathrm{Li}$ atom $\mathrm{D}$-line transitions
}

\author{
RUI Yang, WU YueLong, LI Rui \& WU HaiBin* \\ State Key Laboratory of Precision Spectroscopy, East China Normal University, Shanghai 200062, China
}

\begin{abstract}
We achieve the photon shot-noise limited optical spectroscopy of ${ }^{6} \mathrm{Li}$ cold atoms by developing a heterodyne detection technique, under the condition of extremely low optical power density (below $10^{-3}$ saturation intensity) of probe beam. We establish a transport chain of frequency stability based on an optical frequency comb, and achieve absolute frequencies measurement of $\mathrm{D}_{1}$ lines with an uncertainty of about $1 \mathrm{kHz}$, which is one order of magnitude more accurate than previous measurements. The $2 \mathrm{P}$ fine structure splitting of ${ }^{6} \mathrm{Li}$ and the hyperfine splitting of $2 \mathrm{P}_{1 / 2}$ are $10052.7804(18)$ and 26.1031(14) MHz, respectively, in agreement with recent theoretical calculations. Our results could provide a benchmark to test the theory at the higher precision and help to resolve large discrepancies among previous experiments.
\end{abstract}

cold atoms, absolute frequency measurement, shot noise limit

PACS: 32.10.Fn, 42.62.Eh, 37.10.De, 42.50.Ar

doi: $10.1360 /$ SSPMA-2020-0483 